\title{
Erratum to: Intubation trachéale en médecine d'urgence
}

\section{Erratum to: Tracheal intubation in emergency medicine}

\author{
F. Lenfant \\ (C) SFMU et Springer-Verlag France 2011
}

Erratum to: Ann. Fr. Med. Urgence DOI 10.1007/s13341-011-0043-2.

La référence correcte de l'article de Danguy des Déserts M, Commandeur D, Fourel D, intitulé : Intubation difficile préhospitalière compliquée d'une rupture trachéale (précédemment référencé : Danguy des Déserts $\mathrm{M}$, Commandeur D, Fourel D (2011) Intubation difficile préhospitalière compliquée d'une rupture trachéale. Ann Fr Med Urg 1:89-90) est : Danguy des Déserts M, Commandeur D, Fourel D (2011) Intubation difficile préhospitalière compliquée d'une rupture trachéale. Ann Fr Med Urg 1:136-7.

F. Lenfant $(\bowtie)$

Service d'anesthésie et de réanimation,

université Pierre-et-Marie-Curie,

université Paris-VI, groupe hospitalier Pitié Salpêtrière,

47-83, boulevard de l'Hôpital,

F-75013 Paris, France

e-mail : francois.lenfant@psl.aphp.fr 